\section{REVISTA}

Actualidades Investigativas en Educación

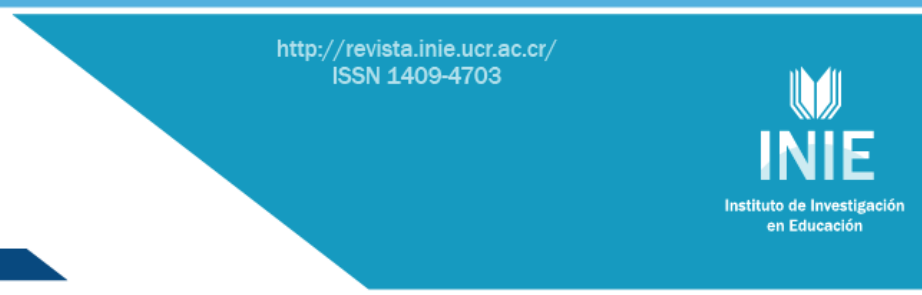

\title{
ACCESO Y USO DE LOS DISPOSITIVOS PORTATILES DE LA POBLACIÓN ESTUDIANTIL DE PRIMARIA A BACHILLERATO: ESTUDIO DE CASO EN ENSENADA, MEXICO
}

ACCESS AND USAGE OF PORTABLE DEVICES OF THE STUDENT POPULATION FROM ELEMENTARY TO HIGH SCHOOL: A CASE STUDY IN ENSENADA, MEXICO.

\section{Volumen 15, Número 3}

Setiembre - Diciembre

pp.1-17

\section{Este número se publicó el $1^{\circ}$ de setiembre de 2015}

DOI: http://dx.doi.org/10.15517/aie.v15i3.20652

Javier Organista Sandoval

Arturo Serrano Santoyo

Revista indizada en REDALYC, $\underline{\text { SCIELO }}$

Revista distribuida en las bases de datos:

CATÁLOGO DE LATINDEX, IRESIE, CLASE, DIALNET, DOAJ, E-REVIST@S, SHERPA/ROMEO, QUALIS, MIAR

Revista registrada en los directorios:

ULRICH'S, REDIE, RINACE, OEI, MAESTROTECA, PREAL, CLACSO 


\title{
ACCESO Y USO DE LOS DISPOSITIVOS PORTATILES DE LA POBLACIÓN ESTUDIANTIL DE PRIMARIA A BACHILLERATO: ESTUDIO DE CASO EN ENSENADA, MÉXICO \\ ACCESS AND USAGE OF PORTABLE DEVICES OF THE STUDENT POPULATION FROM ELEMENTARY TO HIGH SCHOOL: A CASE STUDY IN ENSENADA, MEXICO.
}

\author{
Javier Organista Sandoval ${ }^{1}$ \\ Arturo Serrano Santoyo ${ }^{2}$
}

Resumen. El artículo identifica los principales dispositivos portátiles e Internet que disponen los estudiantes de Educación Primaria, Secundaria y Bachillerato en Ensenada, México y se determina la frecuencia y restricción de su uso en los espacios escolares. Se construyó un indicador de nivel socio-tecnológico (NST) con base en siete variables asociadas con posesiones tecnológicas y servicios en la vivienda para ubicar a estudiantes adscritos a escuelas con NST alto y bajo. Estas variables se recuperaron de la información demográfica publicada por el Instituto Nacional de Estadística y Geografía (INEGI). Se desarrolló una encuesta para recuperar información general de los estudiantes, de sus posesiones tecnológicas, del contexto de uso, entre otros. La encuesta se aplicó a 1972 estudiantes de las escuelas seleccionadas. La posesión de Internet en el NST alto fue mayor al 90\% en todos los niveles, mientras que en el NST bajo de Educación Primaria apenas llega a 57\%. Los resultados muestran una cobertura superior al $90 \%$ en cuanto a computadora y teléfono celular, el mayor porcentaje de posesión de tabletas (46\%) corresponde a estudiantes de Educación Primaria. Al comparar las posesiones tecnológicas de los estudiantes en el NST alto y bajo, se encontraron diferencias significativas $(p<0.05)$ en cuanto a la posesión de equipo de cómputo y tabletas. Se destaca que la mayoría de los estudiantes lleva su teléfono a la escuela, sin embargo, se prohíbe su uso. Los hallazgos muestran que 2 de cada 5 estudiantes cuentan con los tres dispositivos. Esta condición anticipa una generación de estudiantes con mayores recursos tecnológicos y con posibilidad de apoyar sus estudios.

Palabras clave: TECNOLOGÍA EDUCATIVA, CAMBIO TECNOLOGICO, ACCESO A LA INFORMACION, NUEVA TECNOLOGIA, MÉXICO

\begin{abstract}
This research is aimed to identify the usage of portable devices and Internet service of elementary, junior and senior high school students in Ensenada, Mexico. We also investigated the frequency of usage and constraints regarding the use of portable devices in the classroom. We constructed an indicator of sociotechnological level (NST) based on seven variables associated with technological ownership and Internet services in homes to locate students with high and low NST. These variables were recovered from the demographic information published by the National Institute of Statistics and Geography (INEGI) of Mexico from the Population and Housing Census conducted in 2010. A survey was developed to retrieve general information of students, their technology ownership status, and context of usage, among others. The survey consisted of 1972 samples of students from selected schools. The NST indicator related to Internet availability in all academic levels was above $90 \%$, while the NST at the elementary school level was barely $57 \%$. The results show a coverage above $90 \%$ in terms of computer and mobile phone ownership. The highest percentage of ownership of tablets (46\%) corresponds to elementary school students. Technology ownership of students with high and low NST showed significant differences $(p<0.05)$ in terms of computers and tablets, but not for mobile phones. We emphasize that most of the students take their phones to school, but in the majority of cases, their use is prohibited in the classroom. Our findings also show that 2 out of every 5 students have three screens. The possession of technology anticipates a generation of students with more technological resources, which can eventually use them to support their studies.
\end{abstract}

Key words: EDUCATIONAL TECHNOLOGY, TECNOLOGICAL CHANGE, ACCESS TO INFORMATION, NEW TECHNOLOGY, MEXICO

\footnotetext{
Investigador en el área de tecnología educativa. Instituto de Investigación y Desarrollo Educativo, Universidad Autónoma de Baja California, México. Dirección electrónica: javor@uabc.edu.mx

${ }^{2}$ Investigador del Centro de Investigación Científica y de Educación Superior de Ensenada (CICESE), México Dirección electrónica: serrano@cicese.edu.mx
}

Artículo recibido: 5 de diciembre, 2014

Enviado a corrección: 4 de mayo, 2015

Aprobado: 13 de iulio. 2015 


\section{Introducción}

Una característica distintiva de la sociedad occidental en esta segunda década del siglo XXI es su creciente exposición a una amplia gama de dispositivos tecnológicos portátiles. En gran medida, ello se debe al gran desarrollo que han tenido las tecnologías de la información y la comunicación, las cuales han aumentado sus capacidades de procesamiento y de conectividad, además de disminuir los costos de sus productos. Para tener un referente de la cobertura que ya tienen los dispositivos portátiles, la compañía de análisis tecnológicos Gartner señala que desde 2014 la venta anual de smartphones supera la de cualquier otro dispositivo tecnológico en el mundo. Según las proyecciones de dicha compañía, se espera que a corto plazo el mercado de las PC descienda ligeramente mientras que para las tabletas se tenga un crecimiento moderado.

Este panorama tecnológico permite anticipar un nuevo perfil de los estudiantes en edades que van de los 6 a los 18 años y quienes disponen o tienen acceso al menos a un dispositivo portátil. Incluso, es frecuente encontrar a personas que cuentan con las tres pantallas portátiles: laptop, tableta y Smartphone. Esta premisa coincide con los resultados estadísticos presentados en México por el Instituto Nacional de Estadística y Geografía (INEGI, 2013), al señalar que el segmento de población, con edades entre 6 y 17 años, son quienes cuentan con mayor porcentaje de acceso a Internet, equipo de cómputo y teléfono celular.

En este contexto, resulta particularmente interesante y de utilidad pedagógica identificar los tipos de dispositivos portátiles que poseen los estudiantes, la frecuencia con que los usan y las restricciones de uso en los espacios escolares.

\section{Antecedentes}

El gran desarrollo logrado en esta segunda década del siglo XXI por las llamadas Tecnologías de la Información y Comunicación (TIC) ha propiciado que un amplio sector de la población, especialmente los jóvenes, disponga de algún tipo de dispositivo portátil. Un punto de partida es precisar que cuando se menciona a las TIC, estas pueden englobar una amplia diversidad de equipos, servicios y procesos. El carácter polisémico de dicho acrónimo sugiere que en toda investigación que involucre la mediación tecnológica o la inserción de tecnología se especifique el tipo de dispositivo o proceso al que se esté refiriendo.

A esta nueva sociedad, Artopoulos (2011) la denomina la sociedad de las cuatro pantallas (cine, televisión, computadora y teléfono celular) por el impacto que han tenido en 
el desarrollo personal de sus habitantes. En el presente documento se retoma dicho concepto, con la salvedad que las tres pantallas de interés son la laptop, tableta y teléfono celular. Estas tres pantallas, o la combinación de ellas, se anticipa que son el reflejo de las tecnologías que mayormente dispone el estudiante mexicano de la región noroeste del país, y que en gran medida, como señalan Crovi, Garay, López y Portillo (2011), estos dispositivos e Internet son indispensables para los estudiantes en su trayecto escolar, por la diversidad de funciones y herramientas que ofrecen para apoyar las actividades educativas.

Uno de los retos, de acuerdo con Aparici (2011), es que la mayoría de los estudiantes disponen y manejan algún o algunos de los dispositivos portátiles típicos, como laptop, tableta y smartphone, por lo que la comunicación y acceso a la información con mediación de tecnología son parte esencial de su vida diaria. Sin embargo, dentro de las aulas se exponen a modelos pedagógicos antiguos; pero fuera de ellas ponen en práctica modelos basados en la colaboración y la interacción. Esta dualidad entra en franca confrontación con las prácticas educativas tradicionales y con el proceso educativo mismo.

Para el caso de México, ha habido esfuerzos institucionales para dotar de aulas multimedia a los salones de clases, para entregar laptops y tabletas a estudiantes de nivel básico y proporcionar acceso a Internet en los espacios escolares, por mencionar algunas acciones y precisamente, el reto actual es que el docente cuente con una adecuada capacitación para que dichos recursos se utilicen como herramientas pedagógicas.

Este fenómeno de la creciente exposición a las tecnologías portátiles de los estudiantes mexicanos, ha influido en que se implementen acciones gubernamentales para explorar en qué medida se dispone de tales recursos tecnológicos. Así, el Instituto Nacional de Estadística Geografía e Informática (INEGI, 2013) aplicó la Encuesta nacional sobre disponibilidad y uso de las tecnologías de información en los hogares y elaboró el Estudio de hábitos y percepciones de los mexicanos sobre Internet y diversas tecnologías asociadas. Llama la atención que 35\% de los niños y jóvenes entre 6-17 años son usuarios de Internet. Esta información constituye un referente a la presente investigación para dirigir al segmento estudiantil de primaria a bachillerato y de explorar las condiciones de posesión, frecuencia de uso, permisos y la opinión que tienen (autoconcepto) en torno al manejo de los dispositivos portátiles de interés. 
Por su parte, algunas instituciones educativas mexicanas han dirigido investigaciones con el propósito de explorar el nivel de penetración de las Tecnologías de la Información y Comunicación y su impacto pedagógico. Todo ello con la intención de innovar y mejorar la calidad de los procesos educativos. En este sentido, Crovi et al. (2011) realizaron una investigación en tres universidades públicas mexicanas que involucró a estudiantes con edades entre 17 y 24 años. Uno de sus hallazgos es el reposicionamiento de los dispositivos móviles e Internet frente a otras tecnológicas en la comunidad universitaria de estudio. No obstante, los autores señalan que el nivel de apropiación de dichas tecnológicas se ha dado de manera asistemática y con resultados inequitativos. Resultados similares fueron presentados por Aguilar-Barceló y Ramírez-Angulo (2007) para estudiantes universitarios de la región fronteriza de Tijuana México. Estos autores mencionan que el nivel de gasto en tecnologías portátiles de los estudiantes depende del nivel de conocimiento tecnológico que tengan y que las preferencias tecnológicas se dirigen hacia los teléfonos celulares e Internet.

Para Ramírez (2009) el uso educativo de los dispositivos portátiles requiere un análisis a mayor profundidad, ya que dependen en gran medida de la infraestructura de redes inalámbricas existentes. Además, cualquier diseño de materiales educativos debe considerar la capacidad limitada de almacenamiento y lo reducido de las pantallas de los teléfonos celulares. Con relación a este punto, Ramos, Herrera y Ramírez (2010) señalan que el uso pedagógico de recursos tecnológicos portátiles puede convertir cualquier escenario educativo en un ambiente innovador y colaborativo. Fundamental será sustentar estas acciones con teorías y estrategias educativas de acorde al contexto actual.

Con base en lo anterior, se desprende el interés de mostrar los principales hallazgos para identificar las posesiones de dispositivos portátiles (computadora, tableta y teléfono celular) e Internet que disponen los estudiantes de los niveles educativos de primaria, secundaria y bachillerato de la zona urbana de Ensenada B.C. México, así como la frecuencia y restricción de su uso en los espacios escolares. Se recurre a información proporcionada por INEGI, para establecer un patrón de muestreo de los estudiantes con base a un indicador desarrollado a partir de algunas variables de corte socio-tecnológico.

\section{Método}

El presente estudio se aborda desde un enfoque cuantitativo, el cual recurre a una perspectiva metodológica de corte exploratoria-descriptiva. Se utiliza información socioeconómica y tecnológica proporcionada en línea por INEGI del censo 2010 para 
elaborar un indicador de condición socio-tecnológica de las áreas geográficas donde se ubican las escuelas de los estudiantes y así seleccionar a quienes se ubican en escuelas con valores altos y bajos de dicho indicador. Mediante esta estrategia se pretende estimar la magnitud de la diferencia en cuanto a niveles de posesión de dispositivos portátiles según el nivel sociotecnológico. Fundamental fue el uso de aplicaciones en línea como google earth y google map para identificar tales escuelas en la zona de estudio.

3.1. Contexto. El presente estudio se desarrolló en la zona urbana de la ciudad de Ensenada, B.C. México durante el último trimestre de 2013. Para el trabajo de campo se ubicaron escuelas, tanto públicas como privadas, de los niveles primaria, secundaria y preparatoria (bachillerato) de la zona mencionada. La investigación fue posible gracias al financiamiento obtenido de la convocatoria Fondo mixto SEP-IMJUVE 2012 CONACYT de México.

3.2. Selección de los participantes. Un punto de partida fue el desarrollo de una estrategia que permitiera ubicar las escuelas de interés para esta investigación. Para ello, se consideraron variables e indicadores demográficos publicados vía Internet por INEGI con base en el censo de población y vivienda 2010 para la zona urbana de Ensenada México. De las 190 variables e indicadores disponibles se seleccionaron siete, con énfasis en aquellas que propician la posesión y uso de dispositivos portátiles. Estas variables fueron: grado de escolaridad, promedio de ocupantes por vivienda, disposición de servicios públicos, posesión de automóvil, de computadora, de teléfono celular y de Internet. Dichas variables se recodificaron en escala de 0-1 y se sumaron para conformar un indicador de nivel sociotecnológico (NST) para cada una de las áreas geográficas delimitadas bajo el concepto de Área Geo-Estadística Básica (AGEB) de la ciudad de Ensenada (véase fig. 1). 
Fig. 1. Zonificación por AGEB para la zona urbana de Ensenada B.C. México

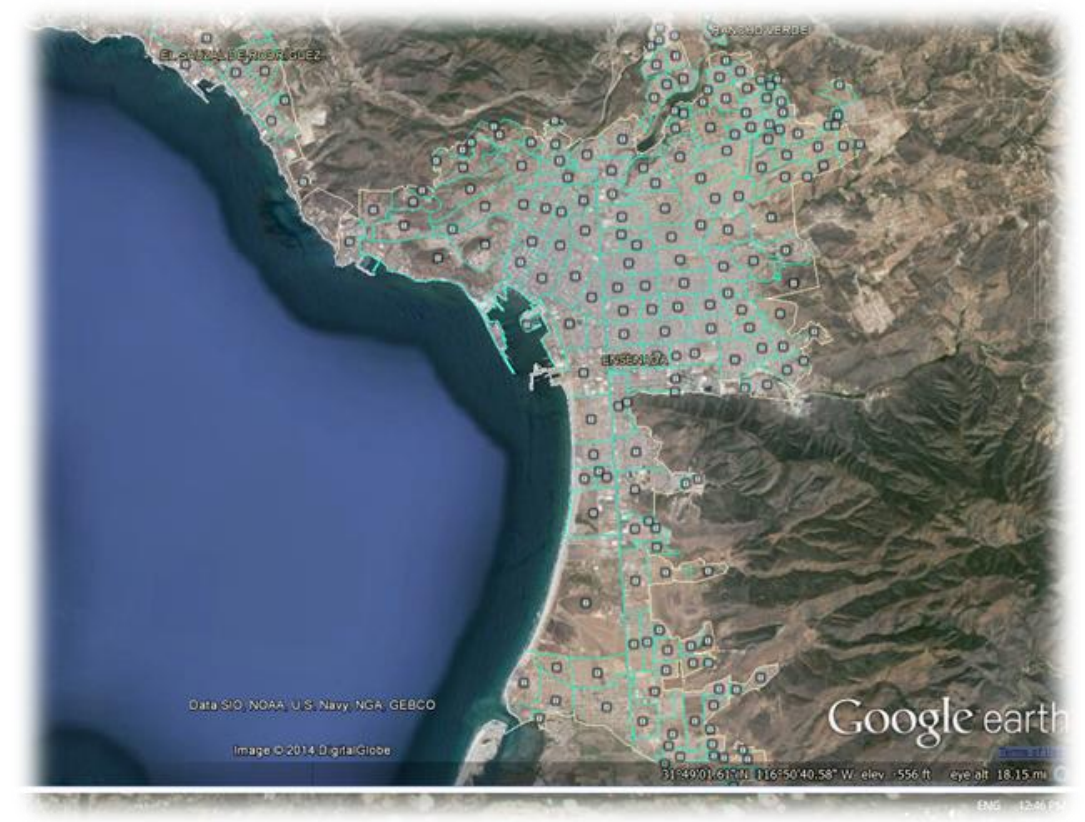

Fuente: Elaboración propia del autor (2014)

Con este indicador calculado para todas las AGEBs de la zona urbana de Ensenada, se seleccionaron escuelas ubicadas en AGEBs con valores máximos y mínimos del indicador NST. La idea tras esta estrategia fue seleccionar estudiantes donde se tuvieran los valores máximo y mínimo de nivel sociotecnológico de sus escuelas de adscripción.

Con base en la información publicada por el sistema educativo de Baja California, México, la matricula estudiantil de la ciudad de Ensenada B. C. para el ciclo escolar 2013-14, se estimó en 127000 estudiantes distribuidos en todos los niveles educativos de dicha zona escolar. El tamaño de muestra obtenido de acuerdo con el algoritmo descrito en Levy y Lemeshow (2008) fue de 2356 alumnos (nivel de confianza 95\%, intervalo de confianza de $2 \%$ ). Se seleccionaron al azar 38 escuelas de la zona urbana y periferia de la ciudad hasta lograr completar la cuota de al menos 600 estudiantes para cada nivel educativo.

La estrategia fue ubicar estudiantes a la mitad de su nivel educativo, ya que se requería que los estudiantes del nivel básico tuvieran habilidades suficientes de comprensión lectora, mientras que en los niveles secundaria y bachillerato se seleccionaron a la mitad de su trayecto escolar para ubicar a quienes tuvieran mayores posibilidades de concluir sus estudios, ya que como señalan Sergio Román y Román (2014), el problema del abandono escolar alcanza cifras de uno de cada dos estudiantes en el nivel medio superior y cercano al 15\% en secundaria. Finalmente se logró recuperar la información de 1972 estudiantes desde 
primaria hasta preparatoria. El segmento estudiantil universitario no se aborda en el presente estudio.

3.3. Instrumentos. Se desarrolló la encuesta de posesión y uso de dispositivos portátiles, la cual tuvo el propósito de recuperar información general de los participantes, de la posesión y frecuencia de uso educativo de los dispositivos portátiles (computadora/laptop, tableta y teléfono celular), tipos de usos educativos realizados con tales dispositivos y algunos aspectos contextuales relacionados con el permiso o prohibición de uso del teléfono celular en espacios escolares. La encuesta consistió de 32 preguntas, organizadas en los siguientes apartados:

- Datos generales de los participantes. Se incluyen variables como edad; género, autoconcepto en manejo de dispositivos portátiles, años de uso, frecuencia de uso, nivel educativo y escuela de adscripción.

- Características técnicas básicas de los celulares. Con énfasis en conectividad, manejo de medios y aplicaciones.

- Condiciones contextuales de uso del teléfono celular. Condiciones de uso del celular en el hogar, en el salón de clases, en la escuela. Permiso y prohibición.

- Limitaciones y beneficios. Opinión de los estudiantes acerca de las principales ventajas y desventajas del teléfono celular en actividades educativas

3.4. Análisis de datos. Se calcularon porcentajes y distribución de ocurrencias. Se obtuvieron estadísticos básicos como media y desviación estándar. Para el caso de las comparaciones se utilizaron los estadísticos de contraste Chi-cuadrado y Kruskal-Wallis.

\section{Resultados}

4.1. Descripción de los participantes. Como parte de la estrategia metodológica de muestreo, se seleccionaron estudiantes cercanos a la mitad de su trayecto escolar para cada nivel educativo. Así, en el nivel primaria se seleccionaron al menos 300 estudiantes del NST bajo y 300 para el NST alto. Las muestras obtenidas se presentan en la tabla 1. 
Tabla 1. Distribución de los participantes según el NST bajo y alto

\begin{tabular}{|l|c|c|c|c|c|c|}
\hline \multirow{2}{*}{} & \multicolumn{2}{|c|}{ NST bajo } & \multicolumn{2}{c|}{ NST alto } & \multicolumn{2}{c|}{ Total } \\
\cline { 2 - 7 } & $\mathrm{n}$ & $\%$ & $\mathrm{n}$ & $\%$ & $\mathrm{n}$ & $\%$ \\
\hline Primaria & 317 & 31.4 & 305 & 31.7 & 622 & 31.5 \\
\hline Secundaria & 376 & 37.3 & 340 & 35.3 & 716 & 36.3 \\
\hline Preparatoria & 316 & 31.3 & 318 & 33.0 & 634 & 32.2 \\
\hline Total & $\mathbf{1 0 0 9}$ & $\mathbf{1 0 0}$ & $\mathbf{9 6 3}$ & $\mathbf{1 0 0}$ & $\mathbf{1 9 7 2}$ & $\mathbf{1 0 0}$ \\
\hline
\end{tabular}

Fuente: Elaboración propia del autor (2014)

4.2. Posesión de dispositivos portátiles e Internet. La figura 2 muestra porcentajes mayores al $90 \%$ en cuanto a la posesión de Internet en el NST alto en todos los niveles educativos. Se destaca que en cada nivel educativo, la diferencia porcentual entre los niveles alto y bajo fue significativa ( $p<0.05$; $U$ Mann-Whitney)

Fig. 2. Porcentaje de posesión de Internet según el NST y nivel educativo

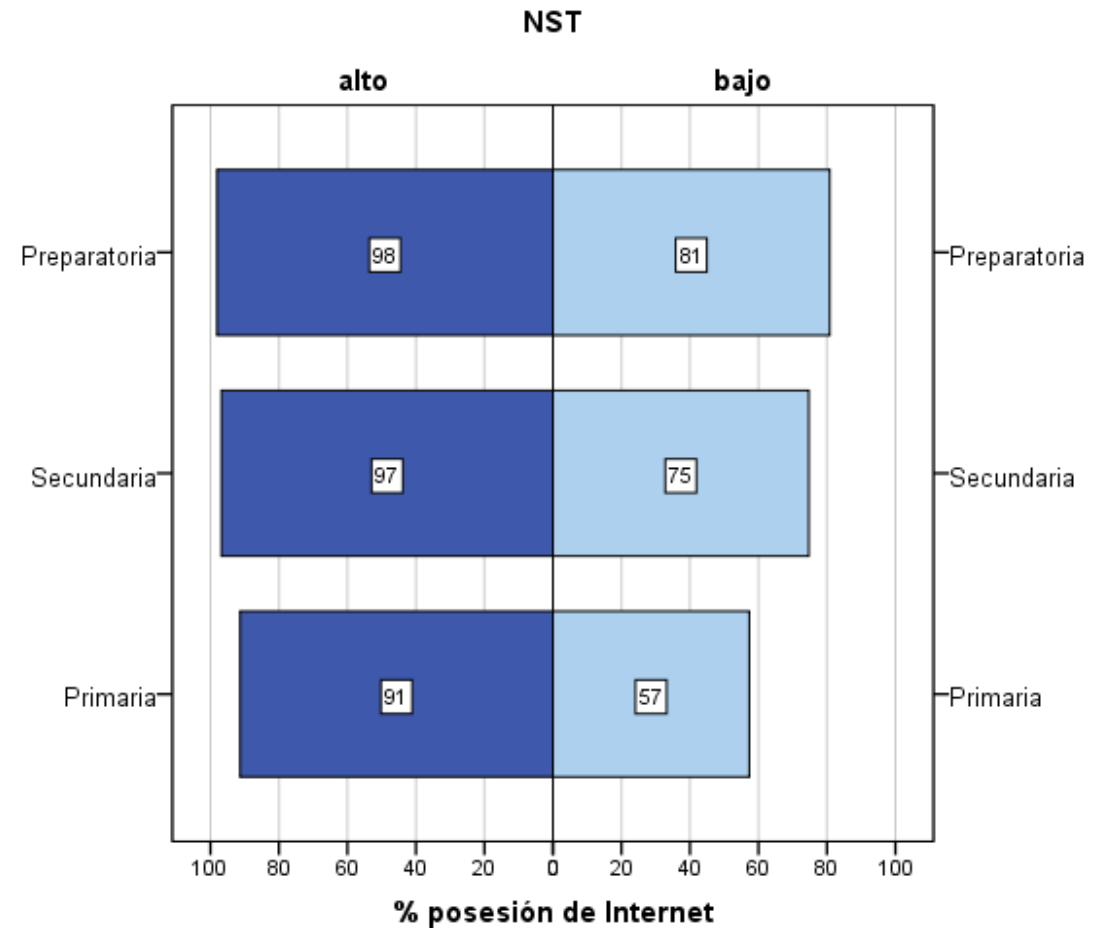

Fuente: Elaboración propia del autor (2014)

- Nivel primaria. Los porcentajes de posesión de computadora, tableta y teléfono celular de los estudiantes de primaria según su NST se muestran en la tabla $2 a$. De manera general, los estudiantes del NST alto cuentan con un mayor porcentaje de dispositivos que los del NST bajo (diferencia significativa al nivel $p<0.05$ ). Llama la atención el alto porcentaje $(59.7 \%)$ de posesión de tabletas en este nivel, superando incluso al 
porcentaje registrado en los niveles secundaria y preparatoria. También se presenta el menor porcentaje de posesión de teléfono celular respecto a los estudiantes de secundaria y preparatoria.

Tabla 2a. Posesión de dispositivos portátiles en el nivel primaria

\begin{tabular}{|c|c|c|c|c|c|c|}
\hline PRIMARIA & \multicolumn{2}{|c|}{ Computadora } & \multicolumn{2}{|c|}{ Tableta } & \multicolumn{2}{|c|}{ Tel. celular } \\
\hline NST & n (\%) & Signif. & n (\%) & Signif. & n (\%) & Signif. \\
\hline Bajo & $\begin{array}{c}201 \\
(63.4 \%)\end{array}$ & 0 & $\begin{array}{c}102 \\
(32.2 \%)\end{array}$ & * * & $\begin{array}{c}164 \\
(51.7 \%)\end{array}$ & 0001 * \\
\hline Alto & $\begin{array}{c}271 \\
(88.9 \%)\end{array}$ & 0.000 & $\begin{array}{c}182 \\
(59.7 \%)\end{array}$ & 0.000 & $\begin{array}{c}200 \\
(65.6 \%)\end{array}$ & 0.001 \\
\hline
\end{tabular}

*Estadístico de contraste Chi-cuadrado. Diferencias significativas al nivel $p<0.05$

Fuente: Elaboración propia del autor (2014)

- Nivel secundaria. Se registran en la tabla $2 b$ diferencias significativas a favor del NST alto en cuanto a la posesión de algún equipo de cómputo (97.9\%) y de tableta (56.8\%). Esta diferencia fue significativa al nivel $p<0.05$. Respecto a la posesión de teléfono celular no se presentaron diferencias significativas entre ambos niveles (NST alto y bajo).

Tabla 2b. Posesión de dispositivos portátiles en el nivel secundaria

\begin{tabular}{|c|c|c|c|c|c|c|}
\hline SECUNDARI & \multicolumn{2}{|c|}{ Computadora } & \multicolumn{2}{|c|}{ Tableta } & \multicolumn{2}{|c|}{ Celular } \\
\hline NST & n (\%) & Signif. & n (\%) & Signif. & n (\%) & Signif. \\
\hline Bajo & $\begin{array}{c}305 \\
(81.1 \%)\end{array}$ & & $\begin{array}{c}106 \\
(28.2 \%)\end{array}$ & & $\begin{array}{c}300 \\
(79.8 \%)\end{array}$ & \\
\hline Alto & $\begin{array}{c}333 \\
(97.9 \%)\end{array}$ & $0.000^{\star}$ & $\begin{array}{c}193 \\
(56.8 \%)\end{array}$ & $0.000^{x}$ & $\begin{array}{c}289 \\
(85.0 \%)\end{array}$ & 0.068 \\
\hline
\end{tabular}

*Estadístico de contraste Chi-cuadrado. Diferencias significativas al nivel $p<0.05$

Fuente: Elaboración propia del autor (2014)

- Nivel preparatoria. Una tendencia similar a la reportada en el nivel secundaria se presentó en preparatoria. Las diferencias significativas (entre NST alto y bajo) se presentaron en cuanto a la posesión de computadora y tableta mas no para teléfono celular (véase tabla 2c.).

Tabla 2c. Posesión de dispositivos portátiles en el nivel preparatoria

\begin{tabular}{|c|c|c|c|c|c|c|}
\hline PREPA & \multicolumn{2}{|c|}{ Computadora } & \multicolumn{2}{|c|}{ Tableta } & \multicolumn{2}{|c|}{ Celular } \\
\hline NST & n (\%) & Signif. & $n(\%)$ & Signif. & n (\%) & Signif. \\
\hline Bajo & $\begin{array}{c}274 \\
(86.7 \%)\end{array}$ & $2^{*}$ & $72(22.8 \%)$ & ○ 0 * & $\begin{array}{c}285 \\
(90.2 \%)\end{array}$ & 0536 \\
\hline Alto & $\begin{array}{c}308 \\
(96.9 \%)\end{array}$ & 0.000 & $\begin{array}{c}179 \\
(56.3 \%)\end{array}$ & 0.000 & $\begin{array}{c}282 \\
(88.7 \%) \\
\end{array}$ & 0.000 \\
\hline
\end{tabular}

${ }^{\star}$ Estadístico de contraste Chi-cuadrado. Diferencias significativas al nivel $p<0.05$

Fuente: Elaboración propia del autor (2014) 
De manera general, en la figura 3 se muestran las tendencias en cuanto a posesión de dispositivos portátiles según el nivel educativo. Se destaca un punto de convergencia en la posesión de algún equipo de cómputo y teléfono celular en los niveles secundaria y bachillerato. El nivel primaria se destaca por tener el mayor porcentaje de posesión de tabletas.

Fig. 3. Porcentaje global de posesión de equipo según el nivel educativo

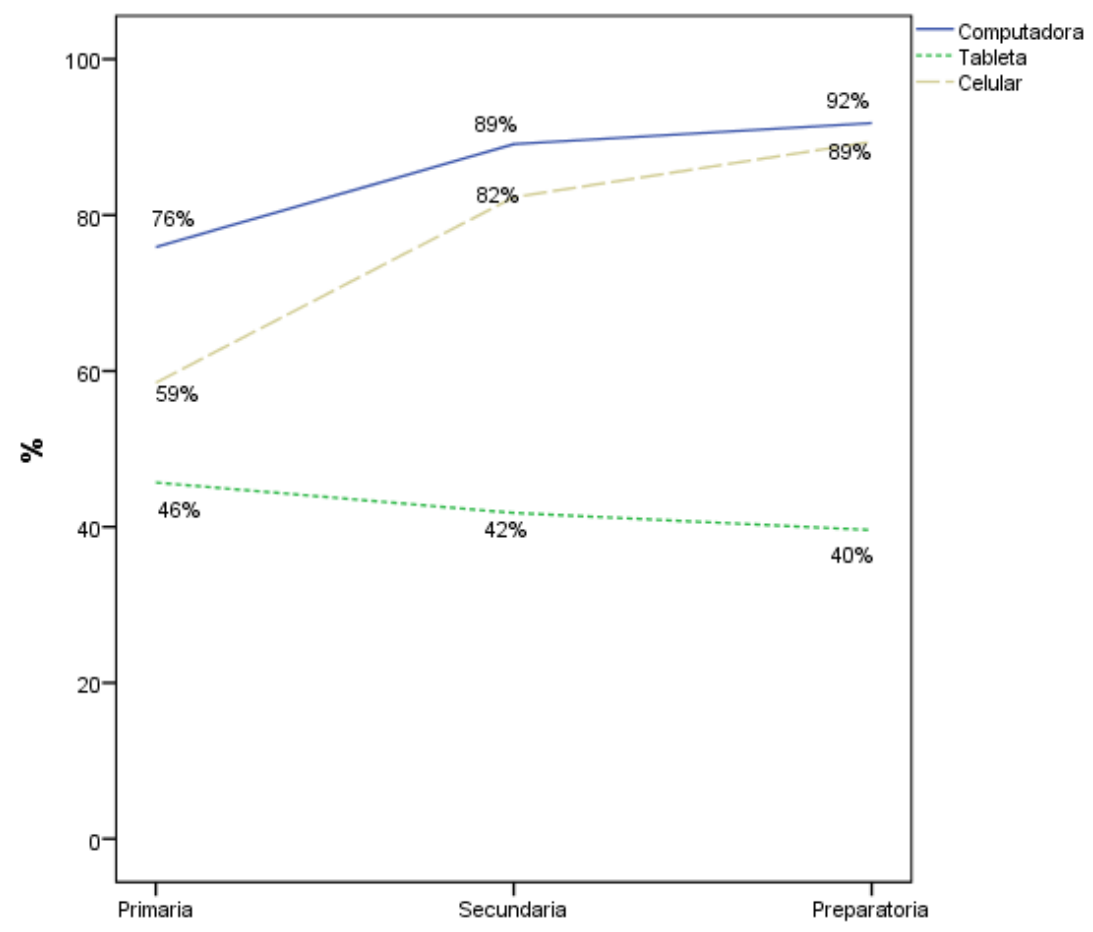

Fuente: Elaboración propia del autor (2014)

La figura 4 muestra el porcentaje de posesión de los tres dispositivos (computadora, tableta y teléfono celular) por parte de los estudiantes según su nivel educativo. Se anticipa una nueva generación de estudiantes que está por ingresar a las universidades y que dispone de las tres pantallas mencionadas. Evidencia de ello es que 2 de cada 5 estudiantes de secundaria y preparatoria cuentan con ellas. 
Fig. 4. Porcentaje de posesión de los tres dispositivos (computadora, tableta y teléfono celular) según el nivel educativo

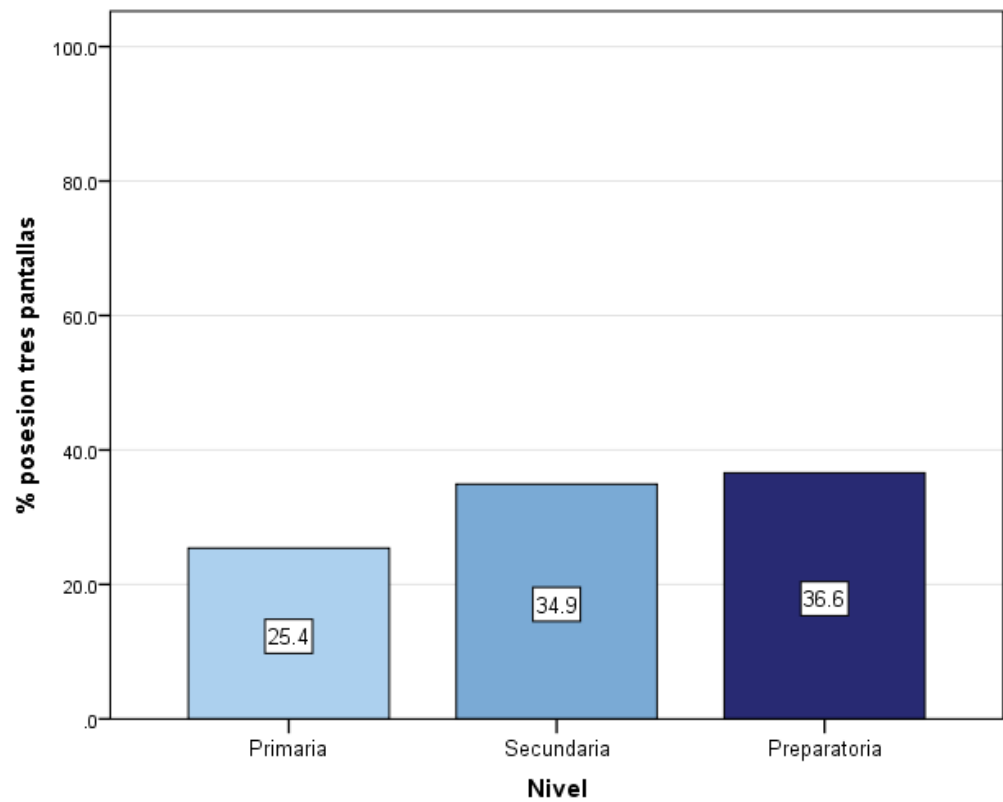

Fuente: Elaboración propia del autor (2014)

4.3. Autoconcepto para manejar el teléfono celular y frecuencia de uso. A la pregunta, ¿Cómo te consideras para manejar el teléfono celular?, en general los estudiantes tuvieron una opinión que muestra valores entre un nivel intermedio y avanzado para manejar tal dispositivo. En lo específico, fueron los estudiantes de secundaria quienes tuvieron la media de opinión más elevada en relación con los otros niveles escolares (diferencias significativas al nivel $p<0.01)$ (ver tabla 3).

Tabla 3. Autoconcepto para manejar el teléfono celular

\begin{tabular}{|l|l|c|c|c|}
\hline & Nivel escolar & Media* & D.E. & $\begin{array}{c}\text { Rango } \\
\text { medio** }^{*}\end{array}$ \\
\hline \multirow{2}{*}{$\begin{array}{c}\text { ¿Cómo te consideras } \\
\text { para manejar el celular? }\end{array}$} & Primaria & 2.3 & 0.94 & 652.9 \\
\cline { 2 - 5 } & Secundaria & 2.6 & 0.65 & 786.2 \\
\cline { 2 - 5 } & Prepa & 2.5 & 0.67 & 747.1 \\
\cline { 2 - 5 } & TOTAL & $\mathbf{2 . 5}$ & $\mathbf{0 . 7 3}$ & \\
\hline
\end{tabular}

${ }^{\star}$ Escala: 0: No familiar, 1: Principiante, 2: Intermedio y 3: Avanzado

${ }^{* *}$ Diferencias significativas entre niveles Primaria, Secundaria y Prepa $(p<0.01)$. Prueba Kruskal-Wallis

Fuente: Elaboración propia del autor (2014)

Para estimar la frecuencia de uso se les preguntó a los estudiantes ¿Cómo cuantas veces al día revisas tu teléfono celular para algo?, los valores medios muestran un incremento de uso 
conforme se aumenta el nivel educativo. Así, la mayor media de uso corresponde a los estudiantes de preparatoria (2.4) lo que sugiere un uso cercano a 40 veces al día. Las diferencias entre niveles escolares fue significativa al umbral $p<0.01$, como se muestra en la tabla 4.

Tabla 4. Frecuencia de uso diario del teléfono celular

\begin{tabular}{|l|l|c|c|c|}
\hline & Nivel escolar & Media & D.E. & $\begin{array}{c}\text { Rango } \\
\text { medio** }^{* *}\end{array}$ \\
\hline \multirow{2}{*}{$\begin{array}{l}\text { Frecuencia* de uso diario } \\
\text { del teléfono celular }\end{array}$} & Primaria & 1.6 & 1.39 & 621.4 \\
\cline { 2 - 5 } & Secundaria & 2.0 & 1.50 & 756.1 \\
\cline { 2 - 5 } & Prepa & 2.4 & 3.58 & 832.8 \\
\cline { 2 - 5 } & TOTAL & $\mathbf{2 . 2}$ & $\mathbf{2 . 2 6}$ & \\
\hline
\end{tabular}

*Escala: $0=[0] ; 1=[1-20] ; 2=[21-40] ; 3=[41-60] 4=[61+]$

${ }^{* *}$ Diferencias significativas entre niveles Primaria, Secundaria y Prepa $(p<0.01)$. Prueba Kruskal-Wallis

Fuente: Elaboración propia del autor (2014)

4.4. Permisos de uso del teléfono celular en la escuela. Para explorar si a los estudiantes se les permite el uso del teléfono celular en los espacios escolares, se les preguntó ¿Llevas el teléfono celular a la escuela? La tabla 5 muestra que los estudiantes de primaria y secundaria lo llevan 'a veces' según lo sugiere el valor medio obtenido, mientras que los de preparatoria prácticamente lo llevan siempre. Estas diferencias en los valores medios fueron significativas al nivel $p<0.01$.

Tabla 5. Estimación de la frecuencia con que llevan el teléfono celular a la escuela

\begin{tabular}{|l|l|c|c|c|}
\hline & Nivel escolar & Media* $^{*}$ & D.E. & $\begin{array}{c}\text { Rango } \\
\text { medio** }^{* *}\end{array}$ \\
\hline \multirow{2}{*}{$\begin{array}{c}\text { ¿Llevas el teléfono } \\
\text { celular a la escuela? }\end{array}$} & Primaria & 0.7 & 0.90 & 448.9 \\
\cline { 2 - 5 } & Secundaria & 1.3 & 1.16 & 655.1 \\
\cline { 2 - 5 } & Prepa & 2.5 & 0.86 & 1060.2 \\
\cline { 2 - 5 } & TOTAL & $\mathbf{1 . 5}$ & $\mathbf{0 . 9 7}$ & \\
\hline
\end{tabular}

*Escala: 0: Nunca, 1: A veces, 2: Muchas veces y 3: Siempre

${ }^{* *}$ Diferencias significativas entre niveles Primaria, Secundaria y Prepa $(p<0.01)$. Prueba Kruskal-Wallis

Fuente: Elaboración propia del autor (2014)

A la pregunta, ¿Te permiten usar el teléfono celular en la escuela?. La tabla 6 muestra valores medios bajos lo que sugiere un alto nivel de restricción en los niveles primaria y secundaria. Por su parte, en preparatoria solo 'a veces' se les permite su uso. Esta diferencia fue significativa al nivel $p<0.01$. 
Tabla 6. Permisos para usar el teléfono celular en la escuela

\begin{tabular}{|l|l|c|c|c|}
\hline & Nivel escolar & Media & D.E. & $\begin{array}{c}\text { Rango } \\
\text { medio** }\end{array}$ \\
\hline \multirow{2}{*}{$\begin{array}{l}\text { ¿Te permiten usar* el } \\
\text { celular en la escuela? }\end{array}$} & Primaria & 0.5 & 0.82 & 687.7 \\
\cline { 2 - 5 } & Secundaria & 0.5 & 0.72 & 660.6 \\
\cline { 2 - 5 } & Prepa & 0.9 & 0.89 & 899.5 \\
\cline { 2 - 5 } & TOTAL & 0.6 & 0.81 & \\
\hline
\end{tabular}

${ }^{\star}$ Escala: 0: Nunca, 1: A veces, 2:Muchas veces y 3:Siempre

${ }^{*}$ Diferencias significativas entre niveles Primaria, Secundaria y Prepa $(p<0.01)$. Prueba Kruskal-Wallis

Fuente: Elaboración propia del autor (2014)

\section{Discusión}

Los hallazgos que aquí se presentan corresponden a una muestra representativa de los estudiantes de primaria, secundaria y preparatoria de la zona urbana de Ensenada, México. La estrategia de muestreo se basó en un indicador de estatus socio-tecnológico, construido a partir de siete variables relacionadas con la disposición de servicios en la vivienda y posesión de dispositivos. Esta información fue recuperada desde el sitio web de INEGI con base en el censo de población y vivienda 2010. Este indicador permitió seleccionar escuelas ubicadas en AGEBs con indicador de NST alto y bajo. Una estrategia similar de construcción de un índice socioeconómico con variables publicadas por INEGI a nivel AGEB fue reportada por Arguelles y García (2013), quienes dan cuenta de la buena aproximación socioeconómica lograda a partir de la utilización de esta información.

En la presente investigación, el supuesto que subyace tras la utilización de variables a nivel AGEB para la construcción de un indicador de nivel socio-tecnológico es que si el estudiante asiste a una escuela ubicada en NST alto, entonces es altamente probable que dicho estudiante disponga de mejores condiciones en cuanto a la posesión de dispositivos portátiles y acceso a los servicios de Internet. Los resultados aportaron evidencia suficiente para corroborar tal supuesto. Los estudiantes ubicados en escuelas con NST alto cuentan con mejores condiciones tecnológicas que aquellos ubicados en escuelas con NST bajo. Estas diferencias fueron significativas al nivel $p<0.05$.

La computadora es un recurso tecnológico que acompaña a la mayoría (90\%) de los estudiantes de los tres niveles aquí considerados. Conforme se incrementa el nivel educativo crece el porcentaje de posesión de este recurso. Por su parte, la tableta encuentra el mayor porcentaje de posesión en la primaria. En gran medida, por ser un recurso tecnológico de bajo precio, amigable y que constituye un primer acercamiento al mundo de la tecnología digital para el estudiante de primaria. Para Hanley (2014) las tabletas son vistas más como 
una forma de entretenimiento pero que difícilmente apoyan la producción de materiales educativos o la elaboración de reportes y tareas por entregar. Son simples dispositivos de consulta de información. Esto coincide con la tendencia encontrada en la presente investigación, en torno a que conforme se aumenta el nivel educativo se incrementa el porcentaje de posesión de algún equipo de cómputo, lo que es un reflejo de la necesidad de los estudiantes de producir contenidos educativos como reportes, ensayos, tareas, etc.

En cuanto al teléfono celular, el porcentaje de posesión muestra una tendencia ascendente, el cual parte de un $60 \%$ en primaria y llega a $90 \%$ en preparatoria. El mayor incremento se presenta de primaria a secundaria. Estos porcentajes coinciden con lo reportado por Cantillo, Roura y Sánchez (2012) para los menores españoles en edades de 10-16 años. De acuerdo con estos autores, el incremento en la posesión de los teléfonos celulares por parte de los niños y jóvenes se debe, en gran medida, a la frecuente exposición al mundo tecnológico y a la creciente necesidad de comunicarse a través de redes sociales y mensajería y de interactuar en espacios creativos.

Es evidente que un estudiante puede tener más de un recurso tecnológico a la vez. Al estimar el porcentaje de estudiantes que cuentan con los tres dispositivos portátiles (computadora, tableta y teléfono celular), los resultados muestran que 2 de cada 5 estudiantes disponen de los tres equipos. Estos resultados dan cuenta del estudiante de las tres pantallas que, a corto plazo, estará incursionando en las aulas universitarias, con suficientes habilidades digitales para apoyar su proceso educativo con esta tecnología.

Con base en la opinión de los estudiantes, se identifica un nivel de manejo de los teléfonos celulares entre los niveles intermedio y avanzado. Estos valores suponen que los estudiantes cuentan con habilidades adecuadas para manejar este tipo de dispositivos. Sin embargo, como sugieren Aparici (2011) y Cantillo et al. (2012), es necesario orientar a los estudiantes en cuanto a las mejores prácticas para explotar este tipo de recursos con un propósito pedagógico.

Se destaca que conforme se incrementa el nivel educativo, también se incrementa la frecuencia con que se transporta el teléfono celular a la escuela. Sin embargo, según se registra en primaria y secundaria, se tiene un nivel alto de prohibición de su uso. Incluso en el nivel preparatoria solamente en ocasiones se permite su uso en los espacios escolares. Estas acciones pueden deberse a la alta capacidad distractora o recreativa que tienen estos dispositivos y se presume que es una de las razones por las que se restringe su utilización en los espacios escolares. 


\section{Conclusión}

Los resultados aquí presentados dan cuenta del contexto escolar de los estudiantes de primaria a bachillerato de una región del noroeste de México, especialmente en cuanto a la posesión de dispositivos portátiles e Internet, su frecuencia de uso y los permisos para su utilización en los espacios escolares. Es notorio que los dispositivos portátiles como laptop, tableta y teléfono celular e Internet se han convertido en recursos tecnológicos que acompañan a la mayoría de los estudiantes de esta región, y son utilizados principalmente en acciones de acceso a información, manejo de comunicación o en aspectos de organización. No obstante, el nivel de posesión de Internet y dispositivos portátiles entre los estudiantes ubicados en NST alto y bajo arrojan diferencias significativas entre ellos, con excepción de teléfono celular en secundaria y preparatoria. Este hallazgo aporta evidencias de que se tiene un segmento de la población estudiantil, de escasos recursos económicos, que no cuenta con un equipo de cómputo, tableta o Internet.

Destaca el hecho de la cobertura casi total de posesión de algún equipo de cómputo, Internet y teléfono celular en el NST alto, lo que sugiere que, al menos la mitad de los estudiantes cuenta con los recursos tecnológicos suficientes para apoyar sus estudios. Inclusive, de manera general, los hallazgos muestran que uno de cada tres estudiantes cuenta con los tres dispositivos (computadora, tableta y teléfono celular) e Internet. Esta condición, abre oportunidades de uso educativo, ya que como señalan Cantillo et al. (2012) los dispositivos portátiles tienen grandes posibilidades educativas para ser utilizados en el aula, ya que favorecen el desarrollo de las competencias básicas.

Se registra en la comunidad estudiantil de esta investigación un contexto de prohibición de uso de los dispositivos portátiles en los espacios escolares, pese a que los estudiantes refieren contar con las habilidades suficientes para manejar tales dispositivos. Esto coincide con lo señalado por Aparici (2011) en el sentido de que la mediación tecnológica es parte esencial de la vida diaria de los estudiantes, pero no dentro de las aulas, lo que sugiere que uno de los retos del sistema educativo está en incorporar los dispositivos portátiles como herramientas pedagógicas. Fundamental será que la comunidad académica cuente con una formación apropiada en el uso de tecnologías, para que sean ellos los promotores- y no los inhibidores- de la mediación tecnológica del proceso educativo.

Los atributos tecnológicos de los dispositivos portátiles que se ofrecen en el mercado, dan cuenta de capacidades como conectividad a Internet, manejo de recursos multimedia, redes de comunicación, descarga de aplicaciones, por mencionar algunas, que pueden 
orientarse hacia un propósito educativo, lo que anticipa que tales dispositivos pueden funcionar como herramientas pedagógicas. Hay un camino por recorrer en cuanto a identificar los factores clave para la inserción exitosa de la tecnología portátil al proceso enseñanza-aprendizaje.

\section{Referencias}

Aguilar-Barceló, José G. y Ramírez-Angulo, Natanael. (2007). Hábitos de consumo de las tecnologías de información en los estudiantes universitarios de Tijuana. Revista Universitaria de la UABC, 5(57), 34-40.

Aparici, Roberto. (2011). Principios pedagógicos y comunicacionales de la educación 2.0. Revista Digital la educ@ción, (145), 1-14. Recuperado de http://www.educoas.org/portal/La Educacion Digital/laeducacion 145/articles/Roberto Aparici.pdf

Arguelles, Karen y García, Luis E. (2013). Construcción de nivel socioeconómico predominante por AGEB mediante análisis multivariado. Milward-Brown. Recuperado de http://blog.amai.org/index.php/construccion-de-nivel-socioeconomico-predominantepor-ageb-mediante-analisis-multivariado/

Artopoulos, Alejandro. (2011). La sociedad de las cuatro pantallas. Una mirada latinoamericana. Barcelona, España: Editorial Ariel, S.A.

Cantillo Velero, Carmen, Roura Redondo, Margarita y Sánchez Palacín, Ana. (2012). Tendencias actuales en el uso de dispositivos móviles en educación. Revista Digital La Educ@ción, 147, 1-21. Recuperado de http://www.educoea.org/portal/La Educacion Digital/147/indexen.html

Crovi, Drueta, Garay Cruz, Delia, López González, Rocío y Portillo Sánchez, Mariela. (2011). Uso y apropiación de la telefonía móvil. Opiniones de jóvenes universitarios de la UNAM, la UACM y la UPN. Revista Derecho a Comunicar, (3), 54-73.

Hanley, Michael. (25 de abril de 2014). Students Prefer Smartphones and Laptops to Tablets, Study Finds. The Chronicle of Higher Education. Recuperado de http://chronicle.com/blogs/wiredcampus/students-prefer-smartphones-and-laptops-totablets-study-finds/51999

Instituto Nacional de Estadística y Geografía - INEGI. (2013). Estadísticas sobre disponibilidad y uso de tecnología de información y comunicación en los hogares, 2013. México: INEGI. Recuperado de http://www.inegi.org.mx/est/contenidos/Proyectos/Encuestas/Hogares/modulos/endutih/ endutih2013/default.aspx

Levy, Paul S. y Lemeshow, Stanley. (2008). Sampling of Populations. Methods and Applications. 4th edition. New Jersey, USA: John Wiley \& Sons, Inc. 
Ramírez Montoya, María Soledad. (2009). Recursos tecnológicos para el aprendizaje móvil (mlearning) y su relación con los ambientes de educación a distancia: implementaciones e investigaciones. Revista Iberoamericana de Educación a Distancia, 12(2), 57-82.

Ramos, Ana Isabel, Herrera, José Alberto y Ramírez, María Soledad. (2010). Desarrollo de habilidades cognitivas con aprendizaje móvil: un estudio de casos. Comunicar, 34, 201209.

Román Morales, Sergio y Román Morales, Luis Ignacio. (2014). MEXICO. Los desafíos de la educación preescolar, básica y media en América Latina. En David Gregosz (Ed.). Los desafíos de educación preescolar, básica y media en América Latina (pp. 207228). Santiago de Chile: KAS/SOPLA. 